\title{
MORSE THEORY FOR G-MANIFOLDS
}

\author{
BY ARTHUR WASSERMAN
}

Communicated by L. Zippin, October 20, 1964

Morse theory relates the topology of a Hilbert manifold [3, §9], $M$, to the behavior of a $C^{\infty}$ function $f: M \rightarrow R$ having only nondegenerate critical points. In applying Morse theory to the study of $G$-manifolds, i.e., manifolds with a compact Lie group $G$ acting as a differentiable transformation group, one must, of course, use maps in the category, i.e., equivariant maps. However, if $x$ is a critical point of an equivariant function then $g x$ is also a critical point for any $g \in G$, hence one must allow critical orbits or, more generally, critical submanifolds.

In $\$ 1$ we give the necessary definitions and notation. In $\S 2$ we extend the results of R. Palais in [3] to study an invariant $C^{\infty}$ function $f: M \rightarrow \boldsymbol{R}$ on a complete Riemannian $G$-space $M$, where in addition to $f$ satisfying condition (C) $[3, \S 10]$, we require that the critical locus of $f$ be a union of nondegenerate critical manifolds in the sense of Bott [1]. In $\S 3$ we show that if $M$ is finite-dimensional then any invariant $C^{\infty}$ function on $M$ can be $C^{k}$ approximated by a $C^{\infty}$ invariant function whose critical orbits are nondegenerate. Together with the results of $\$ 2$ this provides an analogue for $G$-manifolds of the Smale handlebody decomposition technique. Proofs will be given elsewhere.

1. Notation and definition. $G$ will denote a compact Lie group and $M$ a $C^{\infty}$ Hilbert manifold. If $\psi: G \times M \rightarrow M$ is the differentiable action of $G$ on $M$, then, for any $g \in G, \bar{g}: M \rightarrow M$ will denote the map given by $\bar{g}(m)=\psi(g, m) ; \psi(g, m)$ will also be shortened to $g m$. If $M, N$ are $G$-manifolds, then $f: M \rightarrow N$, is equivariant if $f \circ \bar{g}=\bar{g} \circ f$ for all $g \in G$; $f$ is invariant if $f \circ \bar{g}=f$ for all $g \in G$. The tangent bundle $T(M)$ of a $G$-manifold $M$ is a $G$-manifold with the action $g X=d \bar{g}_{p}(X)$, for $X \in T(M)_{p}$. If $E$ and $B$ are $G$-manifolds and $\pi: E \rightarrow B$ is a Hilbert vector bundle [2], then $\pi$ is said to be a $G$-vector bundle if, for each $g \in G, \bar{g}: E \rightarrow E$ is a bundle map. Note that $\pi$ is then equivariant as is the zero-section. If, in addition, $\pi$ has a Riemannian metric, $\langle$,$\rangle ,$ and each $g \in G$ acts isometrically, then $\pi$ will be called a Riemannian $G$-vector bundle. $M$ will be called a Riemannian $G$-space if $T(M) \rightarrow M$ is a Riemannian $G$-vector bundle. Let $f: M \rightarrow R$ be an invariant $C^{\infty}$ function. The gradient vector field, $\nabla f$, on $M$, is defined by $\langle\nabla f, X\rangle$ $=d f_{p}(X)$ for $X \in T(M)_{p}$ and, since $f$ is invariant, $g \nabla f_{p},\langle X\rangle=\left\langle\nabla f_{p}, g^{-1} X\right\rangle$ $=d f_{p}\left(g^{-1} X\right)=d\left(f \circ \bar{g}^{-1}\right)_{o p}(X)=d f_{o p}(X)=\left\langle\nabla f_{o p}, X\right\rangle$ for all $X \in T(M)_{o p}$ 
so $g \nabla f_{p}=\nabla f_{g p}$. Hence, if $\sigma_{p}$ is the maximum solution curve of $\nabla f$ with initial condition $p[3, \S 6]$, then $g \sigma_{p}=\sigma_{g p}$.

At a critical point of $p$, i.e., where $\nabla f_{p}=0$, we have a bounded, self-adjoint operator, the hessian operator, $\phi(f)_{p}=T(M)_{p} \rightarrow T(M)_{p}$, defined by $\left\langle\phi(f)_{p} v, w\right\rangle=H(f)_{p}(v, w)$, where $H(f)_{p}$ is the hessian bilinear form $[3, \S 7]$. A closed invariant submanifold $V$ of $M$ will be called a critical manifold of $f$ if $\partial V=\varnothing, V \cap \partial M=\varnothing$ and if each $p \in V$ is a critical point of $f$. It follows that $T(V)_{p} \subseteq \operatorname{ker} \phi(f)_{p}$, and so there is an induced bounded self-adjoint operator $\bar{\phi}(f)_{p}: T(M)_{p} / T(V)_{p}$ $\rightarrow T(M)_{p} / T(V)_{p}$. If $\bar{\phi}(f)_{p}$ is an isomorphism for each $p \in V$, then $V$ is called a nondegenerate critical manifold of $f$.

Recall that $f$ is said to satisfy condition (C) if each subset $S$ of $M$ on which $f$ is bounded but on which $\|\nabla f\|$ is not bounded away from zero has a critical point of $f$ in its closure.

Definition. The invariant $C^{\infty}$ function of $f: M \rightarrow R$ is called a Morse function for the Riemannian $G$-manifold $M$ if it satisfies condition (C) and if the critical locus of $f$ is a union of nondegenerate critical manifolds without interior.

If $E$ is a Riemannian $G$-vector bundle or Hilbert space then $\|e\|=\langle e, e\rangle^{1 / 2}$ and $E(r)=\{e \in E \mid\|e\| \leqq r\}, E^{\circ}(r)=\{e \in E \mid\|e\|<r\}$ and $\dot{E}(r)=\{e \in E \mid\|e\|=r\}$. If $f: M \rightarrow R$, then $f^{a, b}$ will denote $\{m \in M \mid a$ $\leqq f(m) \leqq b\}$ and $f^{b}=f^{-\infty, b}$.

$C_{G}(M)$ will denote the invariant $C^{\infty}$ functions on the finite-dimensional $G$-manifold $M$ with the $C^{k}$ topology for some fixed $k \geqq 2$. If $f \in C_{G}(M), \epsilon>0$ and $\psi: R^{n} \rightarrow M$ is a coordinate chart for $M$, then a neighborhood of $f$ in the $C^{k}$ topology is given by

$$
\left\{h \in C_{G}(M) \mid N_{k}(f \circ \psi-h \circ \psi)(x)<\epsilon \text { for }\|x\| \leqq 1\right\},
$$

where

$$
N_{k}(f \circ \psi)(x)=\sum_{j=0}^{k}\left\|d^{j}(f \circ \psi)_{x}\right\|,
$$

and \|\| denotes the usual norm on multilinear transformations. $C_{G}(M)$ is a space of the second category.

2. Morse functions. The behavior of a function near a critical manifold is specified by the

MoRse Lemma. Let $\pi: E \rightarrow B$ be a Riemannian $G$-vector bundle and $f$ a Morse function on $E$ having $B$ (i.e., the zero-section) as a nondegenerate critical manifold. If $B$ is compact there is an equivariant diffeomorphism $\theta: E(r) \rightarrow E$ for some $r>0$ such that $f(\theta(e))=\|P e\|^{2}$ $-\|(1-P) e\|^{2}$, where $P$ is an orthogonal bundle projection. 
An important property of Morse functions is given by:

Proposition. If $f$ is a Morse function the critical locus of $f$ in $f^{a, b}$ is the union of a finite number of disjoint, compact, nondegenerate critical manifolds of $f$.

We also have the

DiffEOMORPHISM THEOREM. Let $f$ be a Morse function on $M$ with no critical value in the bounded interval $[a, b]$. If $f^{a-\delta, b+\delta}$ is complete for some $\delta>0$ then $f^{a}$ is equivariantly diffeomorphic to $f^{b}$.

Attaching a handle-bundle.

Definition. Let $V, W$ be Riemannian $G$-vector bundles over $B$. The bundle $V(1) \oplus W(1)=\{(x, y) \in V \oplus W \mid\|x\| \leqq 1,\|y\| \leqq 1\}$ (not a manifold $)$ is called a handle-bundle of type $(V, W)$ with index $=$ dimension of $W$. Let $N, M$ be manifolds with boundary, $N \subset M$ and $f: V(1) \oplus W(1) \rightarrow M$ a homeomorphism onto a closed subset $H$ of $M$. We shall write $M=N \cup_{f} H$, and say that $M$ arises from $N$, by attaching a handle-bundle of type $(V, W)$ if

(i) $M=N \cup H$,

(ii) $f \mid \dot{V}(1) \oplus W(1)$ is a diffeomorphism onto $H \cap \partial N$,

(iii) $f \mid V^{\circ}(1) \oplus W(1)$ is a diffeomorphism onto $M-N$.

Atraching Lemma. Let $\pi: E \rightarrow B$ be a Riemannian G-vector bundle and $P$ an orthogonal bundle projection. Let $V=P(E), W=(1-P)(E)$ and define $f, g: E \rightarrow R$ by $f(e)=\|P e\|^{2}-\|(1-P) e\|^{2}, g(e)=f(e)$ $-3 \epsilon / 2 \lambda\left(\|P e\|^{2} / \epsilon\right)$ where $\epsilon>0$ and $\lambda$ is the function defined in $[3, \S 11]$. Then $\{x \in E(2 \epsilon) \mid g(x) \leqq-\epsilon\}$ arises from $\{x \in E(2 \epsilon) \mid f(x) \leqq-\epsilon\}$ by attaching a handle-bundle of type $(V, W)$.

Note that $B$ is a nondegenerate critical manifold of $f$. By the Morse Lemma we can choose coordinates for $\pi: E \rightarrow B$ such that $f(e)=\|P e\|^{2}$ $-\|(1-P) e\|^{2}$ in a neighborhood of $B$ for any function $f$ having $B$ as a nondegenerate critical manifold. Hence, by abuse of notation, we shall also refer to the handle-bundle of type $(P(E),(1-P) E)$ as the handle-bundle $(B, f)$.

MaIN TheORem. Let $f$ be a Morse function on the complete Riemannian $G$-space $M$. If $f$ has a single critical value $c$ in the bounded interval $[a, b]$, then the critical locus of $f$ in $[a, b]$ is the disjoint union of a finite number of compact submanifolds $N_{1}, \cdots, N_{\mathrm{s}}$. $f^{b}$ is equivariantly diffeomorphic to $f^{a}$ with s handle-bundles of type $\left(N_{i}, f\right)$ disjointly attached.

An excision and Thom's theorem proves the 
Corollary (Bott [1]). Let $N_{1}, \cdots, N_{t}$ be those critical manifolds with index $\left(N_{i}, f\right)=k_{i}<\infty$. Then

$$
B_{m}\left(f^{b}, f^{a} ; Z_{2}\right) \approx \sum_{i=1}^{t} B_{m-k_{i}}\left(N_{i} ; Z_{2}\right) .
$$

Now let $a, b$ be arbitrary regular values of $f, a<b$, and again denote the critical manifolds of finite index $k_{i}$ by $\left\{N_{i}\right\}, i=1, \cdots, t$. Let $R_{m}(X)$ $=$ dimension of $H_{m}\left(X ; Z_{2}\right)$ and $\chi(X)$ the Euler characteristic of $X$. Then we have the Morse inequalities:

(i) $\chi\left(f^{b}, f^{a}\right)=\sum_{i=1}^{t}(-1)^{k_{i}} \chi\left(N_{i}\right)$,

(ii) $R_{m}\left(f^{b}, f^{a}\right) \leqq \sum_{i=1}^{i} R_{m-k_{i}}\left(N_{i}\right)$

(iii) $\sum_{l=0}^{m}(-1)^{m-l} R_{l}\left(f^{b}, f^{a}\right) \leqq \sum_{l=1}^{t} \sum_{l=0}^{m}(-1)^{m-l} R_{l-k_{i}}\left(N_{i}\right)$.

3. Density lemma. Let $M$ be a finite-dimensional $G$-manifold. For any compact subset $A$ of $M, \mathfrak{T}_{G}(A, M) \subset C_{G}(M)$ will denote those functions whose critical locus in $A$ is the union of nondegenerate critical orbits. Clearly $\mathfrak{\pi}_{G}(A, M)$ is open in $C_{G}(M)$.

Lemma 1. Let $G$ act orthogonally on the Euclidean space $V$ with fixed point set $W$. Then $\mathfrak{T}_{G}(W(1), V)$ is open and dense in $C_{G}(V)$.

The proof follows from an application of Sard's theorem to $f \mid W$ (for any $f$ ) and some jiggling of $f$ in the normal direction to $W$. Baire's theorem and a double induction on the dimension and number of components of $M$ yields

Lemma 2. $\mathfrak{N}_{G}(V(1), V)$ is open and dense in $C_{G}(M)$.

One further application of Baire's theorem yields

Density Lemma. For any finite-dimensional G-manifold $M$, $\mathrm{N}_{G}(M, M)$ is dense in $C_{G}(M)$.

Carefully approximating an invariant proper function by a function in $\mathfrak{T}_{G}(M, M)$ gives

Corollary. There exists a Morse function on $M$.

Combining the corollary with the main theorem yields

Corollary. If $M$ is compact then $M=\left(N_{1}, f\right) \cup_{o_{1}}\left(N_{2}, f\right) \cdots$ $\cup_{0_{s}}\left(N_{s}, f\right)$ where the $\left(N_{j}, f\right)$ 's are handle-bundles over orbits.

Vector bundles over orbits can be described as follows: Let $\pi: E \rightarrow \Omega$ be a $G$-vector bundle over the orbit $\Omega, x \in \Omega$ and let $H \subset G$ be the isotropy group of $x$. Then $\Omega \approx G / H$ and $G \rightarrow G / H$ is a principal bundle with structural group $H$. Since $H$ acts linearly on $\pi^{-1}(x)=F$ we have the associated vector bundle $G \times{ }_{H} F$ with fibre $F . G \times{ }_{H} F \rightarrow G / H$ is 
actually a $G$-vector bundle since the actions of $G$ and $H$ on $G \times F$ commute. The projection $G \times F \rightarrow F$ extends by equivariances to a bundle equivalence

$$
\begin{aligned}
& G \times_{H} F \rightarrow E \\
& \downarrow \quad \downarrow \\
& G / H \approx \Omega \text {. }
\end{aligned}
$$

Hence $\pi: E \rightarrow \Omega$ is determined by the action of $H$ on $F$.

\section{REFERENCES}

1. R. Bott, Non-degenerate critical manifolds, Ann. of Math. (2) 60 (1954), 248-261.

2. S. Lang, Introduction to differentiable manifolds, Interscience, New York, 1962.

3. R. Palais, Morse theory on Hilbert manifolds, Topology 2 (1963), 299-340.

BRANDEIS UNIVERSITY

\section{SYMPLECTIC GROUPS OVER DISCRETE VALUATION RINGS}

BY CARL R. RIEHM ${ }^{1}$

Communicated by G. Whaples, October 12, 1964

A symplectic group over a field $\neq F_{2}$ or $F_{3}$, according to a theorem of Dickson and Dieudonné (see [1]), has no normal subgroups other than its center $\{ \pm 1\}$. Attempts at integral analogues of this theorem have of late been quite successful. First Klingenberg [6] showed that every normal subgroup of a symplectic group over a local ring is a congruence group (again with some exceptions). Then Bass, Lazard and Serre [2] showed that every normal subgroup of finite index in the symplectic group $\mathrm{Sp}_{2 n}(Z)$ over the rational integers contains a congruence subgroup if $n \geqq 2$. In [5], Jehne proved local results similar to Klingenberg's, and used them to show that any normal subgroup $G$ of the symplectic group over a suitable Dedekind ring is a congruence subgroup, if $G$ is closed under the congruence topology.

The above three integral results all assumed that the discriminant of the alternating form is a unit. The purpose of this note is to drop this restriction and give a generalization of [6]. In order to obtain a tractable canonical form, it is necessary to assume that the local

\footnotetext{
${ }^{1}$ Research partially supported by National Science Foundation grant GP-1656.
} 